\title{
Li-Fi: A Potential and revolutionary Technology in the field of Visible Light Communication
}

\author{
R.Vishwa ${ }^{1}$, R.Rohith ${ }^{2}$, A.Samuel ${ }^{3}$, A.Sabaresh ${ }^{4}$, P.Sarvesh $^{5}$ \\ 1,2,3,4,5 UG Student, Department of Mechanical Engineering, SNS College of Technology, Coimbatore, Tamil Nadu \\ vishwaraj9566@gmail.com ${ }^{1}$, harirohithsr@gmail.com ${ }^{2}$,robellosam@gmail.com ${ }^{3}$ \\ ashwinsabaresh@gmail.com ${ }^{4}$,sarveshp258@gmiail.com ${ }^{5}$
}

\begin{abstract}
Over 50 billion devices to be connected to the internet.156 million emails, 150,000 Skype calls and over 500,000 comments read and used by every people in a single minute. Most of them are wireless. Radio spectrum is getting full. Did you ever experience a cricket match in the ground? When you want to upload a picture you cannot upload it! Because more people use a single tower they cannot supply faster. There LI-FI kicks in. Instead of using radio waves light waves can be used for communication i.e. LI-FI. Using LI-FI bandwidth becomes very larger and it is more than 1000 times the size of the radio spectrum. This is better and it'll never get full at any time. Light fixture becomes wireless router, gives very fast and very stable connection to the internet. It is faster that you can play 2 HD Movies and a Skype call at the same time. As if now it is the great alternative for Wi-Fi, especially where radio waves are not permitted (or) work poorly. For example: - Hospitals, factories, planes, etc. According to me the main usage of Li-Fi is that light cannot penetrate through walls that give the additional layer of security to banks and more governmental organizations get more secured through this technology.
\end{abstract}

\section{Keywords: Li-Fi, Wi-Fi, Visible light}

\section{Introduction}

In 19th century the wireless communication technology was introduce and the growth of wireless communication technology was huge over the subsequent years. The wireless communication technology acts as the medium of transmitting data from one device to other devices. The air is the channel for transmitting data, it replaces the cables or wires or other electronic conductors. Thereby the channel use electromagnetic waves like IR, RF, satellite, etc. [1-3].The wireless communications technology is influenced most in present world. The wireless communication devices and technologies are ranging from smart phones to computers, tabs, laptops, Bluetooth technology, and printers [4-6] the connecting and communicating between two or more devices is transmitted using wireless signals through the wireless communicating technologies and devices. The main advantage of wireless communication devices is the absence of physical infrastructure. The connection between two or devices are not done by physical connection, which would often include collapsing or space.[7-10] In recent days, because of the unimaginable growth in design and development of technologies, have paved a way for the growth of wireless technology to a greater extent. 


\section{The Visible Light Communication}

Visible Light Communication (VLC) which use only visible light for transmitting data. The speed of light is high that is from 780- 375 nanometres. Mostly modern VLC use digital connections it process data by only 0 s and1s. In ancient times they also used sound waves to transmit the data. Now we almost use radio frequency for everything, sharing data, (e.g) share it, money transaction also takes place by radio frequency Google pay (tez) mode money shared by radio frequency. VLC provide low latency, high bandwidth to transmit more data while radio waves transmit only a limited data because it cannot have more spectrums and more potential for cross talk and interference comparing VLC. It also known for its security feature, which means now technology, has developed to the most but no one has developed to hack a LED Light. Radio waves in atmosphere get disturbed by noise so that the receiver cannot receive good information. But LI-FI can be used over there because it has no problem in the atmosphere and transmission is also high.

Now VLC technologies are more uncommon in every place the communication using light gives increase of strength in wireless devices and radio limited spectrums getting crowded. The VLC gets increased because the technologies are getting more crowded and affect each other in different radio frequencies, does not cause any problem to VLC. Nowadays VLC is used as a communication device to connect millions of people across the world. It is more cost- efficient. Visible Light Communication (VLC) and Optical Wireless Communication (OWC) that use only the intensity of visible light beams and transmit the data. Now it has become advanced and it uses LED and combined with Solid State Lightning (SSL) used as a substitute for radio frequency (RF) gets more benefited.

VLC takes larger visible region for wireless communication in the areas where RF spectrum and radio waves are restricted. Any implementation of new rules (or) facilities is not required. They can also be used in already existing lightning arrestors. They cannot penetrate through the walls so it naturally provides security and it has the capability to avoid the interference from other rooms, thus it provides the already available bandwidth. At present the electronic devices become developed and it contain a photodiode receive signals from light source. Sometimes a cell phone camera or a digital camera is needed. In fact the sensor in the camera is a photodiode (Pixels). In cell phone camera (or) a digital camera a single photodiode is used as a multipurpose object. A sensor may provide multichannel (i.e.) a single pixel can provide a channel or a multiple light source. The block diagram shown in Fig 1.

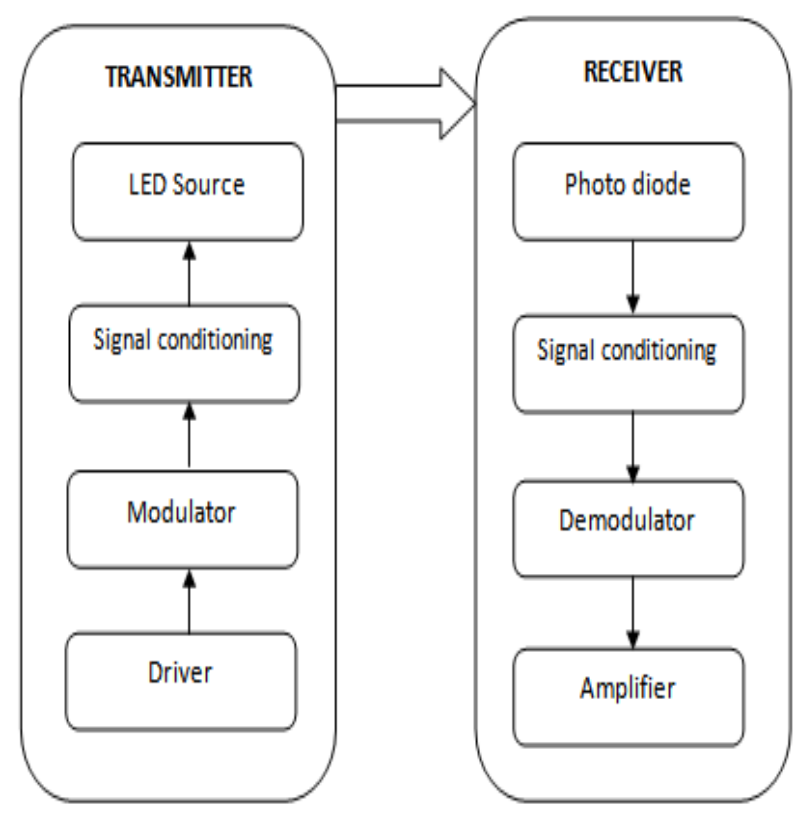

Fig.1 Flowchart of VLC

\section{Li-fi technology}

$\mathrm{Li}-\mathrm{Fi}$ is a visible light communication system (VLC) which stands for Light Fidelity. It runs in the category of wireless communication device and is known for its speed. It was uncovered by Harald Haas a professor in University of Edinburgh revealed it during a TED talk in 2011. He has envisioned light bulbs that could act as a wireless router. $\mathrm{Li}-\mathrm{Fi}$ is expected to solve few of the major concerns faced in implementing 5G. Li-Fi has many favourable advantages over other radio communication technologies Like $\mathrm{Wi}-\mathrm{Fi}$ and Bluetooth. Few to mention are its reliability, data security, and uses light as the medium of data transfer. Li-Fi is seen a as promising technology for interrupted and smooth implementation of $5 \mathrm{G}$ by majority of the telecommunication giants around the globe.

LI-FI is a wireless technology holds key and solve many challenges faced by $5 \mathrm{G}$ such as Frequency Bands, Huge data volume, MIMO Technology and Beam Forming and so on. LI-FI is 
mostly free to use and it is more secure. In present and future it acts vital for $\mathrm{Wi}-\mathrm{Fi}$. It never uses radio frequency for transmission rather it uses light waves for transmission now it gets extremely used by every eco- friendly companies.

\section{Methodology}

$\mathrm{Li}-\mathrm{Fi}$ is a Visible Light Communication (VLC) system. Though $\mathrm{Wi}-\mathrm{Fi}$ and $\mathrm{Li}-\mathrm{Fi}$ are similar in transmission data electromagnetically, Wi-Fi runs on radio waves whereas $\mathrm{Li}-\mathrm{Fi}$ uses visible light waves. Li-Fi has two main components of working, a photo detector and a signal processing element. The data that is transmitted through light is received by the photo detector and is sent to the signal processor. The signal processor in-turn converts the data into readable content. Fig 2 shows process flow chart. The light source of the communication is a semiconductor LED light bulb. This is in added advantage as the current supplied can be reduced or increased at very high speed without being seen by the naked human eye. The data is fed into the LED light bulb and sent at a very high speed which is received by the photo detector. The rise and fall in the LED light is then converted into electrical signal in the receiver. The signal is then converted to the machine readable binary data which can be viewed as video, audio or any other human readable form.

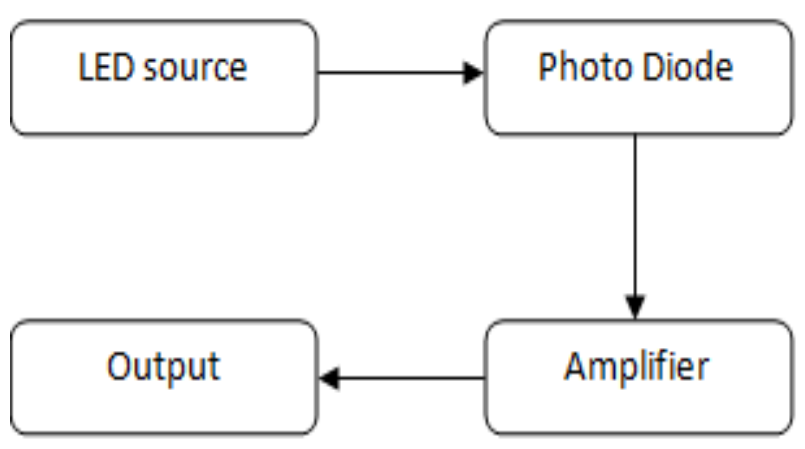

Fig.2 Li-Fi process Flow

Li-Fi uses infrared and visible light spectrum for communicating data at high speed. It reuses the radio frequency spectrum more effectively to achieve higher data rates. The advantage of using visible light over radio waves is that it will not have interference as the radio waves. Each light is driven by a Li-Fi modem or a chip that serves as an access point. As light is spatially confined, it can be used as small cells or packets of information. Though LED are originally the source of illumination, by altering the currents and creating variation, its output can be varied as output data. As typical as other digital devices, led is passed as data 1 whereas the off state is treated as 0 . It is claimed that the rate of data transmission can go up to $10 \mathrm{~GB}$ per second.

There are major companies that have understood the necessity and demand for $\mathrm{Li}-\mathrm{Fi}$ in the future and are taking their parts in the investment. Pure Li-Fi has joined a venture with French lighting company Lucibel to produce Li-Fi enabled products in 2014. There are few usable products that are currently available in the market by PureLi-Fi like Li-Fi ceiling unit and $\mathrm{Li}-\mathrm{Fi}-\mathrm{XC}$. The Li-Fi ceiling unit can be connected to LED fixtures and whereas $\mathrm{Li}-\mathrm{Fi}-\mathrm{XC}$ are much more compatible with other devices using a USB. It is claimed that from each led light that are enabled by Li-Fi, $43 \mathrm{Mbps}$ of data can be provided. Table 1 give comparison between $\mathrm{Li}-\mathrm{Fi}$ and $\mathrm{Wi}-\mathrm{Fi}$. It an interesting space for businesses where $\mathrm{Li}-\mathrm{Fi}$ provides a better and faster connectivity along with data transmission. The Li-Fi provides vast opportunities to the retailer with the integration of internet of things. For example, the data transmission to multiple customers by the shop owners can be done from a distance faster and safer. Along with pure $\mathrm{Li}-\mathrm{Fi}$, the major light and Equipment Company, Philips has also joined hands in contributing its share of $\mathrm{Li}-\mathrm{Fi}$ services. It was also reported that in 2016, Li-Fi was tested in Dubai by the UAE based MNC Du and Zerol. They claimed successful transmission of data in the form of internet, audio, and video using Li-Fi. Li-Fi has already intrigued the ideas of major companies in the field of educational system, medical applications, cheaper internets in aircrafts, underwater application and so on.

Table.1 Comparison of Li-Fi and Wi-Fi

\begin{tabular}{|l|l|}
\hline \multicolumn{1}{|c|}{ Li-Fi } & \multicolumn{1}{|c|}{ Wi-Fi } \\
\hline $\begin{array}{l}\text { Bandwidth } \\
\text { Expansion of Li-Fi } \\
\text { is beyond the limits } \\
\text { of Wi-Fi. }\end{array}$ & $\begin{array}{l}\text { B Bandwidth } \\
\text { Expansion of Wi- }\end{array}$ \\
\hline
\end{tabular}




\begin{tabular}{|c|c|}
\hline $\begin{array}{l}\text { - The speed of the Li- } \\
\text { Fi is } 10 \text { Gbps which } \\
\text { is faster than Wi-Fi. }\end{array}$ & $\begin{array}{l}\text { - The maximum } \\
\text { speed of the Wi-Fi } \\
\text { is } 150 \mathrm{Mbps} \text {. }\end{array}$ \\
\hline $\begin{array}{l}\text { - The data density of } \\
\text { Li-Fi is high }\end{array}$ & $\begin{array}{l}\text { - The data density of } \\
\text { Wi-Fi is low. }\end{array}$ \\
\hline $\begin{array}{l}\text { - The range of Li-Fi is } \\
\text { low but over large } \\
\text { area the } \\
\text { transmission } \\
\text { becomes difficult. }\end{array}$ & $\begin{array}{l}\text { - The range of Wi-Fi } \\
\text { is at medium. }\end{array}$ \\
\hline $\begin{array}{l}\text { - Li-Fi is more secure } \\
\text { when compare to } \\
\text { Wi-Fi. }\end{array}$ & $\begin{array}{l}\text { - Wi-Fi security is } \\
\text { not very good } \\
\text { compared with Li- } \\
\text { Fi. }\end{array}$ \\
\hline $\begin{array}{l}\text { - The power } \\
\text { availability is very } \\
\text { high on Li-Fi. }\end{array}$ & $\begin{array}{l}\text { - The power } \\
\text { availability of Wi- } \\
\text { Fi is lower than Li- } \\
\text { Fi. }\end{array}$ \\
\hline $\begin{array}{l}\text { - The ecological } \\
\text { impact of Li-Fi is } \\
\text { much below the } \\
\text { level of common } \\
\text { wireless devices. }\end{array}$ & $\begin{array}{l}\text { - The ecological } \\
\text { impact is of } \\
\text { - Wi-Fi is not } \\
\text { drastic, but does } \\
\text { have a minimum } \\
\text { significant impact. }\end{array}$ \\
\hline $\begin{array}{l}\text { - The cost required is } \\
\text { very less than } \\
\text { - Wi-Fi. }\end{array}$ & $\begin{array}{l}\text { - The cost of Wi-Fi } \\
\text { is comparatively } \\
\text { higher. }\end{array}$ \\
\hline
\end{tabular}

\section{Challenges and applications}

$\mathrm{Li}-\mathrm{Fi}$ is the latest technology which will offer quickest speed for Internet access. So, it will augment/replace $\mathrm{Wi}-\mathrm{Fi}$ at instructional establishments and at companies in order that the folks there will build use of Li-Fi with the high speed. Operation theatres (OTs) don't enable Wi-Fi thanks to radiation concerns. Usage of $\mathrm{Wi}-\mathrm{Fi}$ at hospitals interferes or blocks the signals for observance equipment. So, it should have unsafe result to the patient's health, thanks to improper working of medical equipment. To beat this and to create OT technical school savvy Li-Fi will be won't to access net and conjointly to manage medical equipment. This can be useful for conducting robotic surgeries and different machine-controlled procedures.

Laptops, sensible phones, tablets and different mobile devices will interconnect directly exploitation Li-Fi. It provides additional security.
The passenger's movement in aircrafts get access to low speed net that too at a really high value. Wi-Fi in tandem with the pilot guidance systems should not be used as a result. For data transfer Li-Fi can be used in aircraft. Li-Fi simply offers an aircraft highspeed network, such as the overhead reading bulb, etc.

Due to efficient signal absorption, the use of RF is unworkable in liquid. The waves are conveyed with small amounts of information. Underwater ROVs are operated by broad cables which supply power and allow pilot signals that are higher than their pilots ' signals to be received. Nonetheless, the link used in ROVs is not enough for exploring wider areas. They could be freer to explore if their wires were replaced by lightweight - say, by a highly powerful submerged lamp. You can use your headlights to communicate, process your data autonomously and sporadically relay your results. $\mathrm{Li}-\mathrm{Fi}$ will even work underwater wherever Wi-Fi fails fully, thereby throwing open endless opportunities for military underwater operations.

Power plants would like quick, inter-connected knowledge systems thus that demand, grid integrity and core temperature (in case of nuclear energy plants) may be monitored. The Radio communication interference is taken into account to be unhealthy for such sensitive areas close these power plants. Li-Fi offers safe, abundant connectivity for all areas of those sensitive locations. Li-Fi may be used as a robust means that of communication in times of disaster like earthquake or hurricanes. Common folks might not apprehend the protocols throughout such disasters. Subway stations and tunnels, common dead zones for most emergency communications, create no obstruction for $\mathrm{Li}-\mathrm{Fi}$.

Though Li-Fi has many advantages when it comes to wireless communication over every other current technology, it has its own challenges which need to be met before implementing it in full scale. For instance, $\mathrm{Li}-\mathrm{Fi}$ requires line of sight of the transmitter and the receiver. Another major question to be answered is the method of transmitting data from the receiver to the transmitter section.

\section{Inference}

In an era of fast communication which has spread to the nook and corner of the world, the systems that 
supports and enables the communication also needs to grow and update. Wireless communication revolutionized the world which was formally controlled by the limitation of wires. Wireless communication paved the way of whole new way and level of communication through different sources like Radio Frequency, infra-Red, Bluetooth communication and so on. Another novel idea which has taken the communication to another level is Li-Fi. Almost all of the limitations that are faced currently by the wireless technologies are beginning to find the answer in the concepts of Li-Fi. The newer and emerging technology under visible light communication tends to make the communication process much simpler and effective in-terms of application and use. It poses a great deal of advantage over other communication technology interms of speed, ease of use, availability of resources and many other features. If implemented in full scale, it even has the potential to change few field drastically for good. Few such fields include education, medical applications, traffic controls, under water communication and so on. Despite of the overwhelming advantages, it too faces few challenges which need to be address to make this technology into ground reality.

\section{Conclusion}

All the street lamps can be transferred to $\mathrm{Li}-\mathrm{Fi}$ lamps to transfer data. As a result of it, it will be possible to access internet at any public place and street. If this technology becomes justifiably marketed then every bulb can be used as a Wi-Fi hotspot to transmit data wirelessly. By virtue of this we can go to a greener, cleaner, safer and a pleasant future. The concept of $\mathrm{Li}-\mathrm{Fi}$ is attracting a lot of eyeballs because it offers a genuine and very efficient alternative to radio based wireless. It has a bright chance to replace the traditional Wi-Fi because as an ever increasing population is using wireless internet, the airways are becoming increasingly obstruct making it more and more difficult to get a reliable, high-speed signal. Li-Fi is the upcoming and on growing technology acting as competent for various other developing and already invented technologies. Hence the future applications of the Li-Fi can be predicted and extended to different platforms and various walks of human life.

\section{References}

[1] S. Vinay Kumar, K. Sudhakar, L. Sudha Rani "Emerging Technology Li-Fi over Wi-Fi" ,International Journal of Inventive Engineering and Sciences (IJIES), 2014.

[2] Sharma, R.R., Sanganal, A., Pati, S. "Implementation of a Simple Li-Fi Based System" International Journal of Computing and Technology 2014.

[3] Sharma, R.R., Raunak, Sanganal, A. " Li-Fi Technology: Transmission of Data Through Light" International Journal of Computer Technology \& Applications 2012.

[4] Little, $T$ "Exploding Interest in Visible Light Communications: An Applications Viewpoint, Smart Lighting" Annual Industry-Academia Days, 2012

[5] Singh, J., Vikash "A New Era in Wireless Technology using Light-Fidelity" International Journal of Recent Development in Engineering and Technology 2014.

[6] Sonnad, A.M., et al., "Recent Advancements in Li-Fi Technology" International Journal of Electrical, Electronics and Data Communication, 2013.

[7] N. Kumar, D. et al., "Visible light communication for intelligent transportation in road safety applications" $7^{\text {th }}$ International Wireless Communication Mobile Computing Conference, 2011.

[8] Jyoti Rani, Prerna Chauhan, Ritika Tripathi, "Li-Fi (Light Fidelity)-The future technology In Wireless communication"”' International Journal of Applied Engineering Research, 2012.

[9] S. Dimitrov and H. Haas "Principles of LED Light Communications: Towards Networked Li-Fi”. Cambridge, U.K.: Cambridge Univ. Press, Mar. 2015.

[10] Jay H. Bhut, Dharmrajsinh N. Parmar, Khushbu V. Mehta, "LI-FI Technology - A Visible Light Communication" International Journal of Engineering Development and Research, 2014. 